\title{
AMP Kinase Activation is Selectively Disrupted in the Ventral Midbrain of Mice Deficient in Parkin or PINK1 Expression
}

\author{
Liting Hang ${ }^{1,2} \cdot$ John Thundyil ${ }^{1} \cdot$ Geraldine W. Y. Goh ${ }^{1} \cdot$ Kah-Leong Lim Li, 2,34 $^{1,4}$
}

Received: 10 September 2018 / Accepted: 1 November 2018 / Published online: 8 November 2018

(c) The Author(s) 2018

\begin{abstract}
Parkinson's disease (PD) is a prevalent neurodegenerative movement disorder that is characterized pathologically by the progressive loss of dopaminergic (DA) neurons in the substantia nigra pars compacta (SNpc) of the midbrain. Despite intensive research, the etiology of PD remains poorly understood. Interestingly, recent studies have implicated neuronal energy dysregulation as one of the key perpetrators of the disease. Supporting this, we have recently demonstrated that pharmacological or genetic activation of AMP kinase (AMPK), a master regulator of cellular energy homeostasis, rescues the pathological phenotypes of Drosophila models of PD. However, little is known about the role of AMPK in the mammalian brain. As an initial attempt to clarify this, we examined the expression of AMPK in rodent brains and found that phosphoAMPK (pAMPK) is disproportionately distributed in the adult mouse brain, being high in the ventral midbrain where the SN resides and relatively lower in regions such as the cortex-reflecting perhaps the unique energy demands of midbrain DA neurons. Importantly, the physiologically higher level of midbrain pAMPK is significantly reduced in aged mice and also in Parkin-deficient mice; the loss of function of which in humans causes recessive Parkinsonism. Not surprisingly, the expression of PGC- $1 \alpha$, a downstream target of AMPK activity, and a key regulator of mitochondrial biogenesis, mirrors the expression pattern of pAMPK. Similar observations were made with PINK1-deficient mice. Finally, we showed that metformin administration restores the level of midbrain pAMPK and PGC- $1 \alpha$ expression in Parkin-deficient mice. Taken together, our results suggest that the disruption of AMPK-PGC-1 $\alpha$ axis in the brains of individuals with Parkin or PINK1 mutations may be a precipitating factor of $\mathrm{PD}$, and that pharmacological AMPK activation may represent a neuroprotective strategy for the disease.
\end{abstract}

Keywords AMP kinase · Mitochondria · Parkinson's disease · Parkin · PGC-1 $\alpha \cdot$ PINK1

Kah-Leong Lim

phslk1@nus.edu.sg

1 National Neuroscience Institute, 11 Jalan Tan Tock Seng, Singapore 308433, Singapore

2 NUS Graduate School for Integrative Sciences and Engineering, National University of Singapore, 21 Lower Kent Ridge, Singapore 119077, Singapore

3 Department of Physiology, Yong Loo Lin School of Medicine, National University of Singapore, 2 Medical Drive, MD9, Singapore 117593, Singapore

4 Neuroscience \& Behavioral Disorders Program, Duke-NUS Medical School, 8 College Road, Singapore 169857, Singapore

\section{Introduction}

Parkinson's disease (PD) is a prevalent neurodegenerative disease that affects millions of predominantly elderly individuals worldwide. Clinically, the disease is attended by a constellation of motoric deficits that includes bradykinesia (slowness in movements), rigidity, and tremor that arise as a result of striatal dopamine depletion due to the progressive loss of midbrain dopaminergic (DA) neurons in the substantia nigra pars compacta $(\mathrm{SNpc})$. Although a subject of intense research, the molecular events underlying PD pathogenesis remain not well understood. Nonetheless, a broad range of studies conducted over the past few decades have implicated aberrant mitochondrial homeostasis as a key culprit (Lim and Zhang 2013). Further supporting this, several groups have found that disease-associated mutations in PD-linked genes, especially Parkin and PINK1, exert 
profound effects on mitochondrial quality control. A corollary to this is that bioenergetics failure may represent a key driver of neurodegeneration in PD (Mattson and Arumugam 2018). This proposition is intuitively logical as post-mitotic neurons particularly those with vast axonal field are known to require high energy to support their operations, which include the active transportation of components (including mitochondria) towards metabolically demanding synaptic terminals that are distally located. In particular, SNpc DA neurons are characterized by unusually large number of axon terminals and axonal arborization, which overall increase their demand for ATP (Pissadaki and Bolam 2013). Indeed, Pacelli and colleagues recently demonstrated that elevated mitochondrial bioenergetics and axonal arborization underlie the selective vulnerability of SNpc DA neurons to degeneration in PD (Pacelli et al. 2015). Thus, strategies that improve the bioenergetics status of DA neurons may potentially be beneficial.

AMP kinase (AMPK) is a key cellular nutrient and energy sensor that is activated in response to falling energy supply, e.g., ATP depletion or glucose starvation (Hardie et al. 2012). It exists as a heterotrimer comprised of a catalytic $\alpha$ subunit and two regulatory subunits: $\beta$ and $\gamma$. AMPK becomes activated upon phosphorylation of the $\alpha$ subunit on threonine-172. The major upstream kinases identified to be responsible for AMPK $\alpha$ Thr172 phosphorylation are liver kinase B1 (LKB1) and calcium/calmodulin-dependent kinase kinase $\beta(\mathrm{CaMKK} \beta)$, although the enzyme can also be activated by pharmacological means via administration of compounds like 5-aminoimidazole-4-carboxamide ribonucleoside (AICAR) and metformin (Zhou et al. 2001). When activated, AMPK switches on catabolic processes that favor energy conservation and restoration, while simultaneously switching off energy-consuming processes (Hardie et al. 2012). Given the critical role of AMPK in regulating intracellular energy metabolism as an adaptive response to energy depletion, it is perhaps not surprising to note that AMPK has profound influence on mitochondrial homeostasis amidst a plethora of metabolic events that it controls. It is well documented that AMPK works through peroxisome proliferator-activated receptor gamma coactivator 1-alpha (PGC-1 $\alpha$ ) to promote biogenesis of mitochondria (Zong et al. 2002). Given this, AMPK activation may represent a suitable strategy to improve the bioenergetic status of DA neurons. Supporting this, we have recently demonstrated that the activation of AMPK mitigates mitochondrial abnormalities and DA neuronal dysfunction in Drosophila genetic models of PD (Ng et al. 2012), and in a PGC-1 $\alpha$-dependent manner ( $\mathrm{Ng}$ et al. 2017). However, whether this strategy works in mammalian models remain to be tested. Notably, little is known to date about the role and regional distribution of AMPK in the mammalian brain. To clarify this, we found here that phospho-AMPK (pAMPK) is regionally distributed in the adult mouse brain in a manner that appears to reflect the unique energy demands of midbrain DA neurons, the expression pattern of which is mirrored by its downstream target PGC- $1 \alpha$. Importantly, the physiologically higher level of midbrain pAMPK is significantly reduced in Parkin-deficient mice, which can be restored by metformin treatment. Similar observations were made with PINK1-deficient mice. Taken together, our results suggest that deficient expression of pAMPK in Parkin or PINK1 mutant midbrains may contribute to bioenergetics impairments in DA neurons and precipitate PD, and that pharmacological AMPK activation may represent a neuroprotective strategy for the disease.

\section{Materials and Methods}

\section{Antibodies}

The following antibodies from Cell Signaling (USA) were used: monoclonal $\alpha$-pAMPK, $\alpha$-tyrosine hydroxylase and polyclonal $\alpha$-TFAM. Antibodies from other manufacturers include monoclonal $\alpha$-AMPK and polyclonal $\alpha$-PGC- $1 \alpha$ (Abcam, UK), monoclonal $\alpha$-PRK8 (Signet/Covance, USA), $\alpha$-beta-actin (Sigma, USA), $\alpha$-PARIS (Merck Millipore, USA), $\alpha$-mouse horseradish peroxidase and $\alpha$-rabbit horseradish peroxidase (GE Healthcare, UK).

\section{Preparation of Mouse Brain Lysates}

All animal-related studies were approved by and conformed to the guidelines of the Institutional Animal Care and Use Committee of TTSH-NNI (TNI-15-01-005). Seven 4-6-month-old wild-type C57B6 male mice or Parkin null mice (Von Coelln et al. 2004) (kind gift from Dr. T. Dawson, Johns Hopkins Medicine), and four 7-month-old PINK1 null mice were sacrificed via cervical dislocation. Male mice only were used in our study as we wish to exclude the potential effects of estrus cycle on AMPK activation, which may

Table 1 Type, age, and sample size of the animals examined

\begin{tabular}{llll}
\hline Figure nos. & Type of mice & Age (months) & Sample size \\
\hline 1 & Wild-type & $4-6$ & 9 \\
2 & Wild-type & $4-6$ & 9 \\
$3 \mathrm{a}$ & Wild-type & 2 & 4 \\
& Wild-type & 20 & 4 \\
$3 \mathrm{~b}$ and c & Wild-type & $4-6$ & 5 \\
$4 \mathrm{a}$ & Parkin null & $4-6$ & 7 \\
$4 \mathrm{~b}$ & PINK1 null & 7 & 4 \\
5 & Parkin null (control) & 9 & 4 \\
& Parkin null (0.1\% met- & 9 & 4 \\
& formin) & & \\
\hline
\end{tabular}


confound our observations. A summary of the age and number of the various mice examined in this study is provided in Table 1. To prepare the lysates, the brain was dissected into different regions (cerebellum, cortex, dorsal midbrain, hippocampus, olfactory bulb, striatum, and ventral midbrain) and homogenized in lysis buffer (PBS with $1 \%$ Triton X-100, cold or $1 \%$ SDS, room temperature, phenylmethylsulfonyl fluoride, aprotinin, Roche, USA), phosphatase inhibitor cocktail 2 and 3 (Sigma, USA) with a plastic homogenizer. Each brain section was placed in a separate microfuge tube. Lysates were sonicated and centrifuged at $13,500 \mathrm{rpm}$ at $4{ }^{\circ} \mathrm{C}$ (for $1 \%$ Triton $\mathrm{X}-100$ ) or $25^{\circ} \mathrm{C}$ (for $1 \%$ SDS) for $15 \mathrm{~min}$ before the supernatant was collected. Protein concentration was quantified using Bradford protein assay and equal amount of lysates was loaded for western blot analysis.

\section{Metformin Treatment of Animals}

Four 5-month-old adult Parkin null male mice were fed with $0.1 \% \mathrm{w} / \mathrm{w}$ metformin feed $\left(\operatorname{Harlan}^{\circledR}\right.$ Labs $0.1 \%$ Metformin Diet) while another group of four age-matched adult Parkin null male mice were fed with control feed (Harlan ${ }^{\circledR}$ Labs Purified Diet) for a period of four months (ad libitum) before they were sacrificed. The brains from these two groups of mice were harvested and dissected for immunoblot analysis according to the methods described above.

\section{Statistical Analysis}

Statistical analyses were performed using the Student's twotailed unpaired $t$-test $\left(* p<0.05,{ }^{* *} p<0.001\right)$. All data were expressed as mean (SEM) generated from at least three independent experiments.

\section{Results}

\section{The Ratio of Phosphorylated AMPK/AMPK is Upregulated in the Ventral Midbrain Region of Normal Mice}

To date, the expression and activity of AMPK in the normal brain remains poorly characterized. To address this, we examined the levels of phosphorylated AMPK (pAMPK) and total AMPK in different regions of the adult mouse brain. Interestingly, we found that the ratio between pAMPK and AMPK differs across various regions of the mouse brain, being significantly higher in the ventral midbrain region relative to the cortex and other regions (Figs. 1, 2a). Given that the ventral midbrain contains nigral DA neurons, it is tempting to think that its high pAMPK/AMPK ratio may reflect the well-known high
A

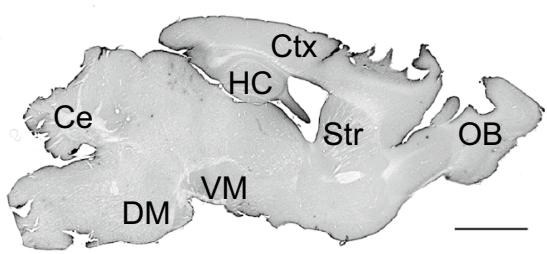

B

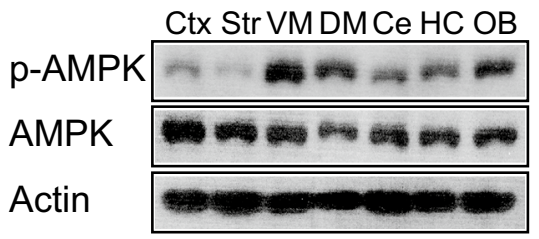

C

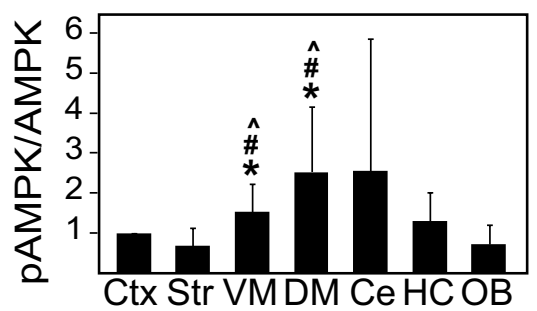

Fig. 1 Phosphorylated AMPK/AMPK ratio varies across the different brain regions of normal mice. a Sagittal section of wild-type mouse brain depicting the location of the respective dissected brain regions (Ctx cortex, Str striatum, $V M$ ventral midbrain, $D M$ dorsal midbrain, $C e$ cerebellum, $H c$ hippocampus, $O B$ olfactory bulb), scale bar = $2 \mathrm{~mm}$. b Immunoblots showing the levels of pAMPK and AMPK across the different brain regions prepared from 4- to 6-month-old wild-type mice. The blots above were stripped and reprobed with anti-actin antibody to reflect loading variations. c Bar graph showing the average densitometric value of $\mathrm{pAMPK} / \mathrm{AMPK}$ ratio in these regions $(n=9)$. $\left({ }^{*} p<0.05\right.$ compared to $\mathrm{Ctx},{ }^{\#} p<0.05$ compared to Str, $\wedge^{p}<0.05$ compared to $\left.\mathrm{OB}\right)$

energy demands of this group of neurons. However, the phenomenon may be confined to the cell body of DA neurons as the pAMPK/AMPK ratio is comparatively reduced in striatal regions where the terminals of these neurons reside (Figs. 1, 2a). Consistent with this profile, the level of PGC- $1 \alpha$, a downstream target of AMPK, correlates well with the level of AMPK activity in the different brain regions (Fig. 2b). As AMPK is also known to promote the expression of nuclear-encoded mitochondrial proteins through the activation of transcription factors, we measured the expression of TFAM and found that the level of this important nuclear-encoded mitochondrial transcription factor is similarly elevated in the ventral midbrain region relative to other regions examined (Fig. 2c). As age is an unequivocal risk factor for the development of PD, we also examined whether the level of AMPK activity in the brain may be affected by the aging process. Interestingly, although we found that the pAMPK/AMPK 

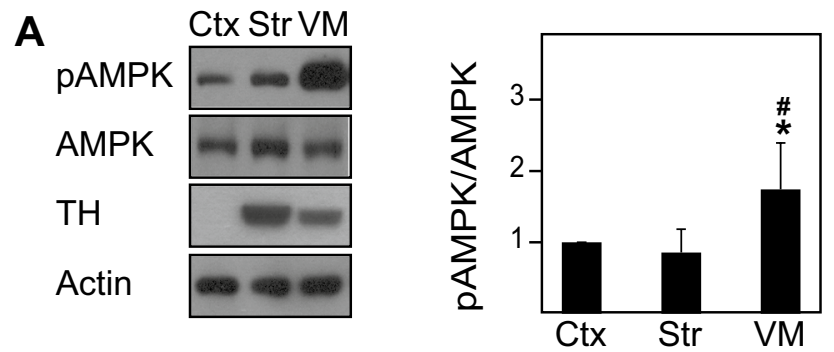

B
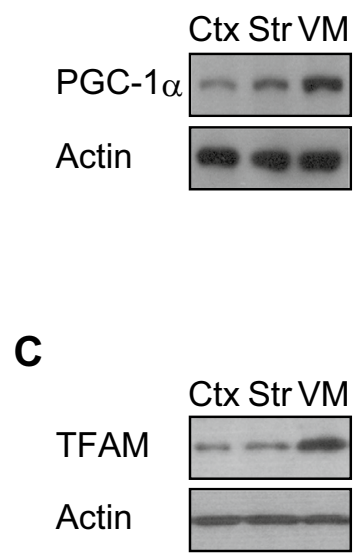
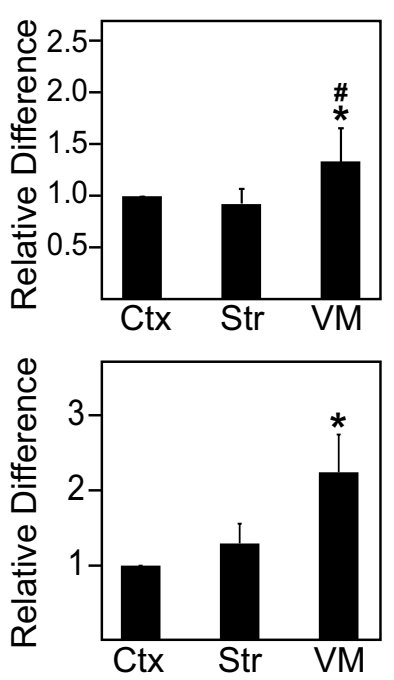

Fig. 2 Phosphorylated AMPK/AMPK ratio, PGC- $1 \alpha$, and TFAM expression are significantly upregulated in the ventral midbrain region. a Left, immunoblots showing the levels of pAMPK, AMPK, and tyrosine hydroxylase (TH) in three selected regions (i.e., Ctx, Str, and VM) of the wild-type mouse brain. Right, bar graph showing the average densitometric value of pAMPK/AMPK ratio in these regions $(n=9)$. b Same as a except that PGC- $1 \alpha$ expression is shown here $(n=5)$. $\mathbf{c}$ Same as a except that TFAM expression is shown here $(n=5)$. The blots above were stripped and reprobed with anti-actin antibody to reflect loading variations. $(* p<0.05$ compared to $\mathrm{Ctx}$, ${ }^{\#} p<0.05$ compared to Str)

ratio exhibits a general trend of reduction with age, the decrease is selectively significant in the ventral midbrain of 20-month-old mice relative to their younger 2-monthold counterparts (Fig. 3a).

\section{The Expression of Phosphorylated AMPK is Significantly Reduced in the Ventral Midbrain of Aged, Parkin-Deficient, and PINK1-Deficient Mice}

Our previous report demonstrating that AMPK activation mitigates the pathological phenotypes of Drosophila Parkin null mutants (Ng et al. 2012) suggests that AMPK and Parkin may be functionally connected. To further investigate the potential relationship between AMPK and Parkin, we examined the expression of Parkin in cortical, striatal, and ventral midbrain regions harvested from normal mouse brain and found that Parkin expression correlates with the levels of pAMPK
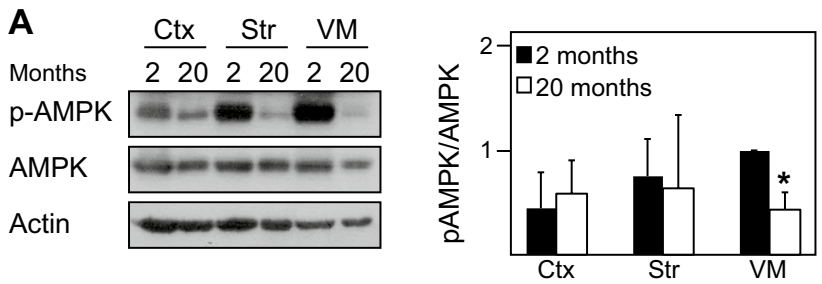

B
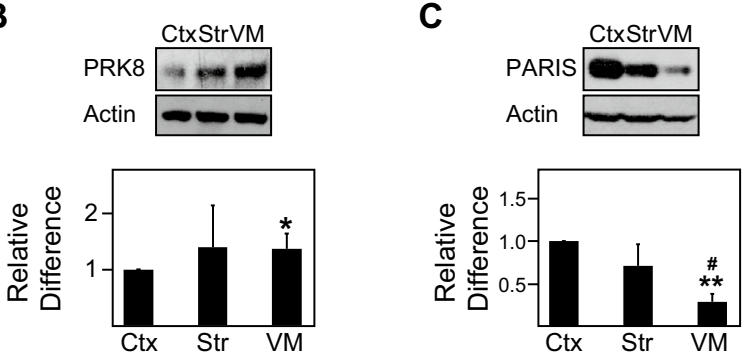

Fig. 3 Phosphorylated AMPK/AMPK ratio is significantly reduced in the ventral midbrain region of aged wild-type mice. a Left, immunoblots showing the levels of pAMPK and AMPK in the same selected brain regions of aged wild-type mice ( 20 month old, $n=4)$ compared to young wild-type mice ( 2 month old, $n=4$ ). Right, bar graph showing the average densitometric value of pAMPK/AMPK ratio in these regions. b Top, immunoblots showing the levels of Parkin in same selected brain regions of wild-type mice. Bottom, bar graph showing the average densitometric value of Parkin levels in these regions $(n=5)$. c Same as b except that PARIS expression is shown here $(n=5)$. The blots above were stripped and reprobed with anti-actin antibody to reflect loading variations. $(* p<0.05, * * p<0.001 \mathrm{com}$ pared to $\mathrm{Ctx},{ }^{\#} p<0.05$ compared to $\left.\mathrm{Str}\right)$

(Fig. 3b), which is interesting. Correspondingly, the expression of PARIS, a negative regulator of PGC- $1 \alpha$ transcription, in the ventral midbrain is reverse that of pAMPK and Parkin (Fig. 3c), which may also explain the observed enhanced PGC-1 $\alpha$ level in this region (Fig. 2b). Importantly, when we repeated the analysis with brain regions derived from Parkin null mice (4-6 months old), we recorded a significant and selective decrease in the pAMPK/AMPK ratio in the ventral midbrain region of these mutant mice (Fig. 4a). Alongside this, we also observed an associated significant reduction in the level of PGC-1 $\alpha$ in the ventral midbrain region of Parkindeficient mice relative to their control counterparts (Fig. 4a). Consistent with the report by Shin et al. (2011), we found that PARIS expression is upregulated in the ventral midbrain of these mutant mice (Fig. 4a). As Parkin and PINK1 functions are intimately interwoven, we also examined the above phenomena in PINK1-deficient mice. We found that PINK1deficient mice, like their Parkin counterparts, exhibit selective 


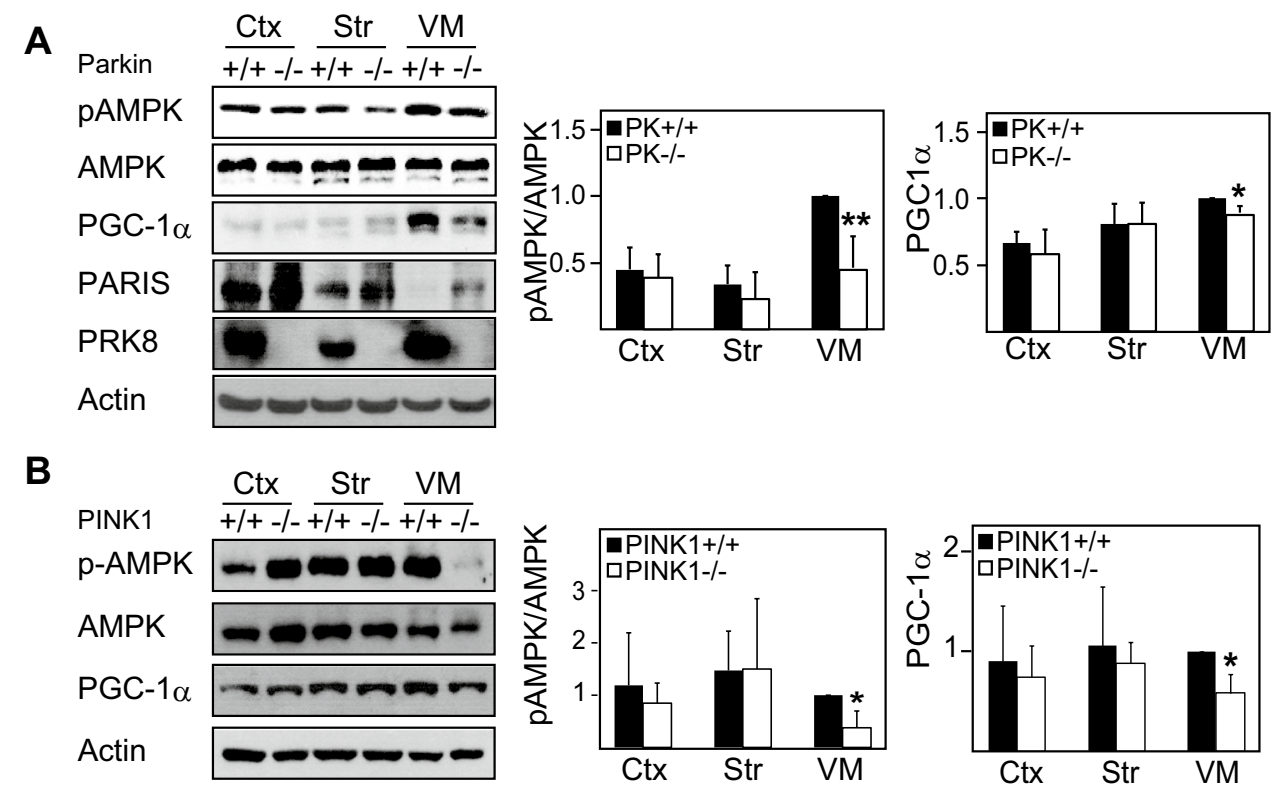

Fig. 4 Phosphorylated AMPK/AMPK ratio is significantly reduced in the ventral midbrain region of Parkin null or PINK1 null mice brain. a Left, immunoblots showing the levels of pAMPK, AMPK, PGC- $1 \alpha$, PARIS, and Parkin in the three selected brain regions of 4-6-month-old wild-type (+/+) and Parkin null (-/-) mice. Right, bar graphs showing the average densitometric value of pAMPK/ AMPK ratio and PGC- $1 \alpha$ level in these regions $(n=7)$. (B) Left, immunoblots showing the levels of pAMPK, AMPK, and PGC-1 $\alpha$ in

reduction in the pAMPK/AMPK ratio as well as PGC- $1 \alpha$ expression in their ventral midbrain region (Fig. 4b). Taken together, our results indicate a selective impairment of the AMPK-PGC-1 $\alpha$ axis in Parkin- and PINK1-deficient ventral midbrain neurons.

\section{The Reduced Ratio of Phosphorylated AMPK/AMPK in the Ventral Midbrain Region of Parkin-Deficient Mice is Rescued by Metformin Treatment}

Given the observed reduction in the levels of pAMPK and PGC- $1 \alpha$ in the midbrain of Parkin-deficient mice, we wished to find out whether pharmacological activation of AMPK could restore their deficiency. We therefore administered Parkin null mice with metformin (an AMPK activator) via oral feed. Consistent with a report by Martin-Montalvo et al. (2013), metformin treatment for a period of 4 months increases AMPK activity in the liver of these mice, suggesting that metformin has been effectively metabolized, although no significant changes in body mass were recorded, other than a slight increase in mass in the metformin-treated group at 2-month post-treatment (not shown). Importantly, treatment of these mice with metformin results in a dramatic and significant rescue of the level of phosphorylated AMPK that is otherwise deficient in the ventral midbrain region the three selected brain regions of 7-month-old wild-type $(+/+)$ and PINK1 null (-/-) mice. Right, bar graphs showing the average densitometric value of pAMPK/AMPK ratio and PGC- $1 \alpha$ level in these regions $(n=4)$. The blots above were stripped and reprobed with antiactin antibody to reflect loading variations. All the experiments above were repeated at least three times. $(* p<0.05$ and $* * p<0.001$ compared to respective wild-type region)

of Parkin null mice (Fig. 5). Curiously, we documented an increased level of PARIS in the metformin-treated group relative to their untreated counterparts (Fig. 3). Notwithstanding this, we found that the level of PGC- $1 \alpha$ is enhanced in the ventral midbrain region of metformin-treated Parkin null mice (Fig. 5), suggesting that pharmacological AMPK activation alone is sufficient to restore the deficient AMPKPGC-1 $\alpha$ pathway in Parkin null mice.

\section{Discussion}

As an initial attempt to clarify the role of AMPK in the mammalian brain, we have conducted a regional expression profiling of pAMPK and AMPK in the brains of normal and Parkin/PINK1 null mice in the present study. In agreement with published literature documenting the high metabolic activities of SNpc DA neurons, we found that the pAMPK/ AMPK ratio (which reflects the cellular energy requirements) is significantly higher in the ventral midbrain of the rodent brain compared to various other regions examined. Interestingly, this physiological upregulation of midbrain pAMPK/AMPK ratio is selectively downregulated in Parkin and PINK1 null mice. Not surprisingly, the expression of PGC- $1 \alpha$, a downstream target of AMPK, essentially follows 
Fig. 5 Metformin treatment selectively restores midbrain pAMPK/AMPK in Parkin null mice. Immunoblots show the levels of pAMPK, AMPK, PGC- $1 \alpha$, and PARIS in the ventral midbrain (VM) tissues of 9-month-old Parkin null mice in the absence or presence of metformin treatment $(n=4)$. Bar graphs show the average densitometric value of pAMPK/ AMPK ratio, PGC- $1 \alpha$, and PARIS in untreated and treated Parkin null mice $\left({ }^{*} p<0.05\right.$ compared to control diet)
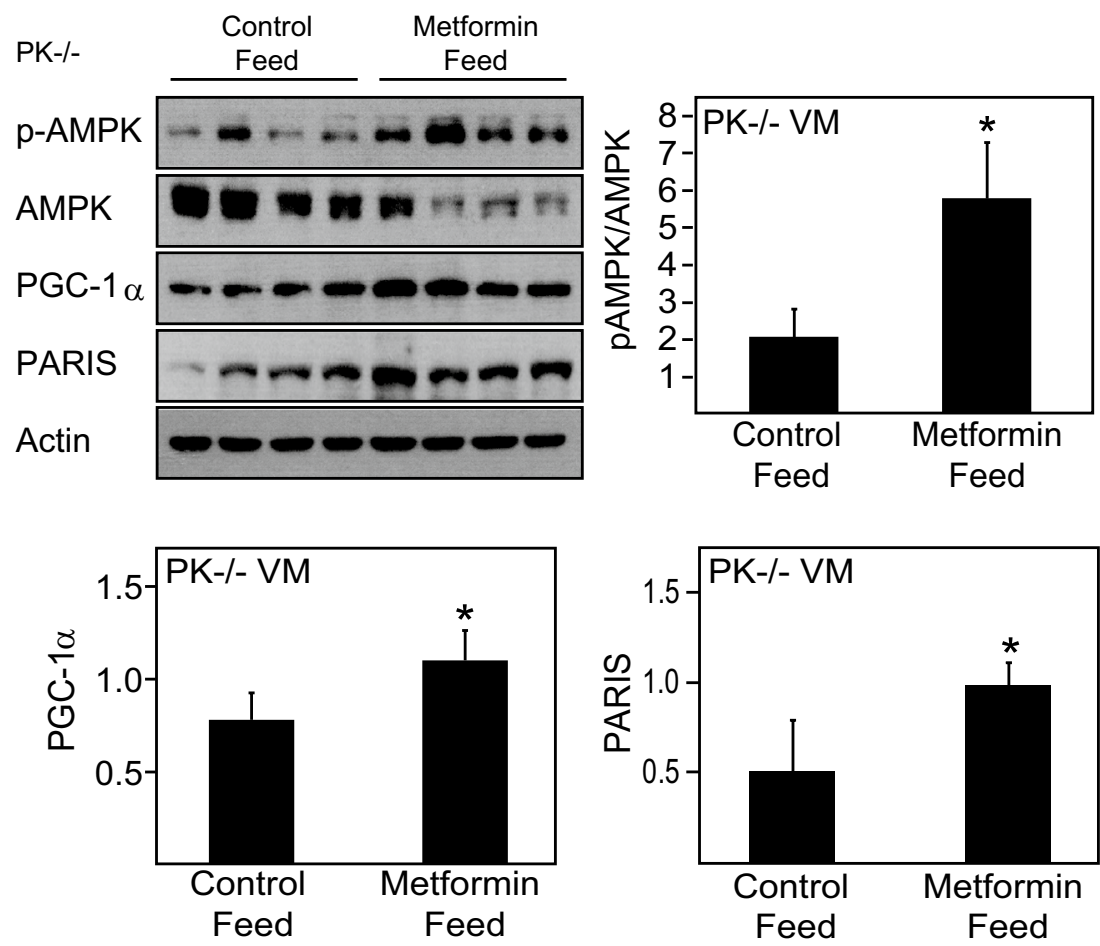

the profile of phosphorylated AMPK. Although somewhat preliminary, it is tempting to suggest from our findings here that impaired AMPK/PGC- $1 \alpha$ activation may contribute to DA neurodegeneration in Parkin- and PINK1-related PD cases, particularly in view of recent reports highlighting that SNpc DA neurons are characterized by unusually high energy demands (Pacelli et al. 2015; Pissadaki and Bolam 2013). However, we are cognizant that neither Parkin nor PINK1 null mice exhibit robust signs of Parkinsonism despite showing selective disruption of AMPK-PGC- $1 \alpha$ axis in their ventral midbrain. It is likely that a combination of extrinsic and intrinsic factors is involved in mediating overt neuronal death, as we and others have previously documented (Zhang et al. 2017; Sulzer 2007), and that AMPK deficiency represents one of these contributing factors to PD pathogenesis.

It is noteworthy to highlight that several groups have found that AMPK activation could confer neuroprotection in PD models. For example, Choi and colleagues demonstrated that AMPK is activated in mice treated with MPTP and that inhibition of AMPK function by compound $\mathrm{C}$ enhances MPP(+)-induced cell death (Choi et al. 2010). Similar findings were made in another recent study albeit in cultured cells exposed to rotenone (Wu et al. 2011). However, in contradiction to these findings, Kim et al. found that AMPK mediates the atrophy of DA neurons in mice exposed to 6-hydroxydopamine (6-OHDA) (Kim et al. 2013). In a related study, $\mathrm{Xu}$ et al. observed similar detrimental effects of AMPK activation in primary neurons treated with
6-OHDA, MPP+, or rotenone (Xu et al. 2014). Thus, the role of AMPK in neuroprotection remains controversial. Notwithstanding this, our own study conducted previously in Drosophila genetic models of PD demonstrating that AMPK activation ameliorates the pathological phenotypes of the mutant flies would favor a neuroprotective function of AMPK. Furthermore, a cohort-based study involving 800,000 individuals revealed that Metformin-inclusive sulfonylurea therapy significantly reduces the risk for the disease occurring with Type 2 diabetes in a Taiwanese population (Wahlqvist et al. 2012), suggesting that AMPK activation is beneficial for PD. Related to this, a recent system of biology-based study identified PGC- $1 \alpha$ as a potential therapeutic focus for intervention in PD (Zheng et al. 2010). Notably, the authors found that bioenergetic genes responsive to PGC- $1 \alpha$ are under-expressed in patients with $\mathrm{PD}$, suggesting that the upregulation of PGC- $1 \alpha$ may be beneficial.

Given that AMPK is a potent upstream activator of PGC-1 $\alpha$, it is intuitive to propose that pharmacological enhancement of AMPK-PGC-1 $\alpha$ activity would represent a rational therapeutic strategy. Interestingly, Martin-Montalvo et al. recently demonstrated that prolonged treatment of mice with metformin significantly improves both their lifespan and health span, although the brain AMPK profile was not examined (Martin-Montalvo et al. 2013). Using similar treatment paradigm, we showed that metformin administration promotes the activation of AMPK (and concomitantly PGC- $1 \alpha$ expression) selectively in ventral midbrain region of Parkin null mice that effectively restore the otherwise 
deficient pAMPK level in these mutant mice. Interestingly, we observed that the upregulation of AMPK-PGC- $1 \alpha$ pathway took place against the backdrop of an enhanced PARIS expression in the metformin-treated group. Although PARIS functions as a negative regulator of PGC- $1 \alpha$ expression, pharmacological AMPK activation alone is clearly sufficient to circumvent the repression by PARIS to result in the restoration of the deficient AMPK-PGC- $1 \alpha$ axis in Parkin null mice. Overall, our finding extended the study by Martin-Montalvo et al. (2013) and provides support to the above-mentioned Taiwanese cohort study that documented a reduced risk for metformin-treated diabetic patients to develop PD (Wahlqvist et al. 2012).

In conclusion, the main finding of our current study is that the AMPK activation is physiologically upregulated in the mammalian ventral midbrain and selectively reduced in the same region in the absence of Parkin or PINK1, the deficiency of which may contribute to SNpc DA neurodegeneration in Parkin/PINK1-related PD cases. Alongside this, we also found that the deficiency in midbrain AMPK expression may be corrected by pharmacological treatment with AMPK activators.

Acknowledgements This work was supported by Grants from the National Medical Research Council: Translational Clinical Research Program in Parkinson's disease and Collaborative Basic Research Grant (CBRG12nov034) (LKL). Ms. Hang L is supported by a Graduate Scholarship from the National University of Singapore Graduate School for Integrative Sciences and Engineering.

\section{Compliance with Ethical Standards}

Conflict of interest The authors declare that they have no conflict of interest.

Open Access This article is distributed under the terms of the Creative Commons Attribution 4.0 International License (http://creativeco mmons.org/licenses/by/4.0/), which permits unrestricted use, distribution, and reproduction in any medium, provided you give appropriate credit to the original author(s) and the source, provide a link to the Creative Commons license, and indicate if changes were made.

\section{References}

Choi, J. S., Park, C., \& Jeong, J. W. (2010). AMP-activated protein kinase is activated in Parkinson's disease models mediated by 1-methyl-4-phenyl-1,2,3,6-tetrahydropyridine. Biochemical and Biophysical Research Communication, 391(1), 147-151. https:// doi.org/10.1016/j.bbrc.2009.11.022.

Hardie, D. G., Ross, F. A., \& Hawley, S. A. (2012). AMPK: A nutrient and energy sensor that maintains energy homeostasis. Nature Reviews Molecular Cell Biology, 13(4), 251-262. https://doi. org/10.1038/nrm3311.

Kim, T. W., Cho, H. M., Choi, S. Y., Suguira, Y., Hayasaka, T., Setou, M., et al. (2013). (ADP-ribose) polymerase 1 and AMP-activated protein kinase mediate progressive dopaminergic neuronal degeneration in a mouse model of Parkinson's disease. Cell Death and Disease, 4, e919. https://doi.org/10.1038/cddis.2013.447.

Lim, K. L., \& Zhang, C. W. (2013). Molecular events underlying Parkinson's disease-An interwoven tapestry. Frontiers in Neurology, 4, 33. https://doi.org/10.3389/fneur.2013.00033.

Martin-Montalvo, A., Mercken, E. M., Mitchell, S. J., Palacios, H. H., Mote, P. L., Scheibye-Knudsen, M., et al. (2013). Metformin improves healthspan and lifespan in mice. Nature Communications, 4, 2192. https://doi.org/10.1038/ncomms3192.

Mattson, M. P., \& Arumugam, T. V. (2018). Hallmarks of brain aging: Adaptive and pathological modification by metabolic states. Cell Metabolism, 27(6), 1176-1199. https://doi.org/10.1016/j. cmet.2018.05.011.

Ng, C. H., Basil, A. H., Hang, L. T., Tan, R., O’Neill, S., Zhang, X., et al. (2017). Genetic or pharmacological activation of the Drosophila PGC-1 $\alpha$ ortholog spargel rescues the disease phenotypes of genetic models of Parkinson's disease. Neurobiology of Aging. https://doi.org/10.1016/j.neurobiolaging.2017.03.017.

Ng, C. H., Guan, M. S., Koh, C., Ouyang, X., Yu, F., Tan, E. K., et al. (2012). AMP kinase activation mitigates dopaminergic dysfunction and mitochondrial abnormalities in Drosophila models of Parkinson's disease. Journal of Neuroscience, 32(41), 1431114317. https://doi.org/10.1523/JNEUROSCI.0499-12.2012.

Pacelli, C., Giguere, N., Bourque, M. J., Levesque, M., Slack, R. S., \& Trudeau, L. E. (2015). Elevated mitochondrial bioenergetics and axonal arborization size are key contributors to the vulnerability of dopamine neurons. Current Biology, 25(18), 2349-2360. https ://doi.org/10.1016/j.cub.2015.07.050.

Pissadaki, E. K., \& Bolam, J. P. (2013). The energy cost of action potential propagation in dopamine neurons: Clues to susceptibility in Parkinson's disease. Frontiers in Computational Neurosciences, 7, 13. https://doi.org/10.3389/fncom.2013.00013.

Shin, J. H., Ko, H. S., Kang, H., Lee, Y., Lee, Y. I., Pletinkova, O., et al. (2011). PARIS (ZNF746) repression of PGC-1alpha contributes to neurodegeneration in Parkinson's disease. Cell, 144(5), 689-702. https://doi.org/10.1016/j.cell.2011.02.010.

Sulzer, D. (2007). Multiple hit hypotheses for dopamine neuron loss in Parkinson's disease. Trends in Neuroscience, 30(5), 244-250. https://doi.org/10.1016/j.tins.2007.03.009.

Von Coelln, R., Thomas, B., Savitt, J. M., Lim, K. L., Sasaki, M., Hess, E. J., et al. (2004). Loss of locus coeruleus neurons and reduced startle in Parkin null mice. Proceedings of National Academy of Science of USA, 101(29), 10744-10749. https://doi.org/10.1073/ pnas.0401297101.

Wahlqvist, M. L., Lee, M. S., Hsu, C. C., Chuang, S. Y., Lee, J. T., \& Tsai, H. N. (2012). Metformin-inclusive sulfonylurea therapy reduces the risk of Parkinson's disease occurring with Type 2 diabetes in a Taiwanese population cohort. Parkinsonism and Related Disorders. https://doi.org/10.1016/j.parkreldis.2012.03.010.

Wu, Y., Li, X., Zhu, J. X., Xie, W., Le, W., Fan, Z., et al. (2011). Resveratrol-activated AMPK/SIRT1/autophagy in cellular models of Parkinson's disease. Neurosignals, 19(3), 163-174. https://doi. org/10.1159/000328516.

Xu, Y., Liu, C., Chen, S., Ye, Y., Guo, M., Ren, Q., et al. (2014). Activation of AMPK and inactivation of Akt result in suppression of mTOR-mediated S6K1 and 4E-BP1 pathways leading to neuronal cell death in in vitro models of Parkinson's disease. Cellular Signaling, 26(8), 1680-1689. https://doi.org/10.1016/j.cells ig.2014.04.009.

Zhang, C. W., Tai, Y. K., Chai, B. H., Chew, K. C. M., Ang, E. T., Tsang, F., et al. (2017). Transgenic mice overexpressing the divalent metal transporter 1 exhibit iron accumulation and enhanced Parkin expression in the brain. Neuromolecular Medicine, 19(23), 375-386. https://doi.org/10.1007/s12017-017-8451-0.

Zheng, B., Liao, Z., Locascio, J. J., Lesniak, K. A., Roderick, S. S., Watt, M. L., et al. (2010). PGC-1alpha, a potential therapeutic 
target for early intervention in Parkinson's disease. Science Translational Medicine, 2(52), 52ra73. https://doi.org/10.1126/scitr anslmed.3001059.

Zhou, G., Myers, R., Li, Y., Chen, Y., Shen, X., Fenyk-Melody, J., et al. (2001). Role of AMP-activated protein kinase in mechanism of metformin action. Journal of Clinical Investigation, 108(8), $1167-1174$.
Zong, H., Ren, J. M., Young, L. H., Pypaert, M., Mu, J., Birnbaum, M. J., et al. (2002). AMP kinase is required for mitochondrial biogenesis in skeletal muscle in response to chronic energy deprivation. Proceedings of National Academy of Sciences of USA, 99(25), 15983-15987. https://doi.org/10.1073/pnas.252625599. 